\title{
Minimizing the risk of COVID-19 among patients on dialysis
}

\author{
T. Alp Ikizler $\mathbb{B}^{1 凶}$ and Alan S. Kliger ${ }^{2}$
}

Patients on haemodialysis or peritoneal dialysis are likely to be at increased risk of novel coronavirus disease (COVID-19). Preventive strategies must be implemented to minimize the risk of disease transmission in dialysis facilities, including education of staff and patients, screening for COVID-19 and separation of infected or symptomatic and non-infected patients.

Novel coronavirus disease (COVID-19) caused by severe acute respiratory syndrome coronavirus 2 (SARS-CoV-2) was designated a global pandemic on 11 March 2020 by the World Health Organization (WHO). Up-to-date epidemiological data show the extent of global infection ${ }^{1}$. The symptoms that are associated with COVID-19 are non-specific and include fever (44-98\% of patients), dry cough ( $68-76 \%$ of patients), muscle pain ( $18 \%$ of patients) and fatigue ( $18 \%$ of patients $)^{2}$. Initial data on infectivity suggest a maximum likelihood of reproductive number for SARS-CoV-2 of 2.8 (that is, a person with the disease is likely to transmit it on average to a maximum of 2.8 other people if no one in their community is immune). This estimate will likely change as more data are analysed. Reported mortality data vary widely from $2.3 \%$ in China to $7.2 \%$ in Italy, where the highest number of patients has been diagnosed to date ${ }^{3}$.

Patients on maintenance dialysis are likely to be at increased risk of COVID-19 and its complications because they tend to be of older age and to have multiple co-morbid conditions, often including hypertension, and suppressed immune systems. As yet, no large-scale reports exist of mortality data in this vulnerable population. The need for frequent travel to dialysis units and unavoidable patient clustering during dialysis shifts further increase the risk of viral transmission. Appropriate preventive strategies must therefore be rapidly employed to minimize the risk of COVID-19 among patients on haemodialysis or peritoneal dialysis in outpatient facilities ${ }^{4}$.

Although much of the available data on COVID-19 are in the form of preliminary reports based on small patient numbers, certain basic principles for prevention and control of communicable diseases can be implemented in this time of crisis (BOX 1). The US Centers for Disease Control and Prevention (CDC) has published interim guidance for the prevention and control of COVID-19 in outpatient haemodialysis facilities that can be freely accessed online $e^{5,6}$ and several dialysis organizations have also developed interim guidance for physicians.

\section{Education}

Education about COVID-19 is required for health-care workers and patients. Crucially, the basics of hand and respiratory hygiene as well as coughing etiquette must be emphasized. When educating patients, special attention should be given to using terminology that is consistent with their health literacy and avoiding medical jargon. Health-care workers should be educated in the selection and use of personal protective equipment (PPE) and should practise putting it on and taking it off correctly. Continuous education efforts are key to preventing health-care worker-related transmission of COVID-19 (REF.7).

\section{Screening of patients}

Best practice for detection of early infection includes phoning all patients before each dialysis shift to determine whether they have COVID-19 symptoms. This approach is dependent on staff availability, and implementation must be facility specific. Every facility must direct symptomatic or COVID-19-exposed patients to a designated screening location before they enter the facility waiting room. Face masks and hand sanitizers must be provided and patients kept at least 6 feet $(\sim 2 \mathrm{~m})$ apart from each another. Each facility should develop a site-specific triage plan to transfer patients to appropriate testing clinics, hospitals or designated COVID-19 dialysis units, if available.

\section{Management of infected patients}

Currently, many dialysis facilities direct symptomatic or COVID-19-exposed patients to hospitals to minimize exposure of uninfected patients in dialysis facilities; however, hospital resources are likely to rapidly reach capacity, with full emergency departments, inpatient beds, intensive care units and inpatient dialysis facilities. Outpatient dialysis facilities must anticipate this likelihood and develop protocols and equipment to manage patients with confirmed or suspected COVID-19.

Patients with COVID-19 symptoms or illness must wear appropriate face masks on arrival and at all times 


\section{Box 1 | Prevention and control of COVID-19 infection in dialysis facilities}

Education of patients and health-care workers

- Hand and respiratory hygiene and coughing etiquette

- Use of personal protective equipment (PPE)

\section{Screening of patients}

- Phone all patients or advise all patients to phone before each dialysis shift to determine whether they have novel coronavirus disease (COVID-19) symptoms

- Place signs to direct symptomatic or exposed patients to a designated screening location in an appropriate space

- Implement triage protocol and provide face masks if applicable

Managing patients with symptoms or illness

- Patients with symptoms or illness should wear appropriate face masks when in the dialysis facility

- If possible, dialyse patients with COVID-19 symptoms or illness in a separate room from patients who are not thought to be infected

- If multiple patients are infected, cohort to separate those with COVID-19 from those who are not thought to be infected

- Separate patients by 6 feet $(\sim 2 \mathrm{~m})$ in all directions

- Assign a selected group of health-care workers to patients who have symptoms or illness; these workers should follow standard contact and droplet precautions with eye protection

- Maintain routine cleaning and disinfection procedures

Management of resources

- Keep track of PPE inventory

- Prioritize use of PPE to conserve stocks

- Consider extended use of eye and face protection

- Consider recycling of PPE and alternative PPE production methods

Management of the workforce

- Use the workforce efficiently

- Create back-up lists and cross-train staff so that positions can be filled if health-care workers become sick

- Protect health-care workers who are not working from potential exposure so that they can provide back-up if necessary when in the dialysis facility. Some dialysis organizations mandate use of face masks for every patient at every dialysis session but this approach has important resource implications and is not currently recommended by the CDC. Patients on home dialysis should be handled with the same protocols as those on in-centre dialysis when they attend outpatient clinics.

Some dialysis organizations are developing cohort models to localize patients who are symptomatic or COVID-19 positive who might be transferred or admitted to different facilities to separate them from those who are asymptomatic or COVID-19 negative. When such arrangements are not available, patients with COVID-19 symptoms or illness should be grouped together in a specific section of the unit and dialysed during the same shift, preferably the last of the day. Ideally, they should be dialysed in a separate room from uninfected patients with the door closed. If a separate room is not available, foot traffic around these patients must be minimized by using corner or end-of-row stations. Symptomatic patients should be spaced at least 6 feet $(2 \mathrm{~m})$ apart in all directions. No requirement exists for patients with COVID-19 to be treated in an airborne infection isolation room. Furthermore, hepatitis B isolation rooms should not be used for patients with COVID-19 unless the facility does not have any patients on haemodialysis with hepatitis B infection.

A selected group of health-care workers should be assigned to patients with COVID-19 symptoms or illness and should follow standard contact and droplet precautions with eye protection (goggles or face shields). Routine cleaning and disinfection procedures are appropriate for COVID-19 in dialysis settings. Staff performing these functions should use the same PPE as those delivering dialysis treatments.

\section{Management of resources}

The possibility of a prolonged pandemic raises the very real threat of equipment shortage and supply chain deficiencies. Tracking of PPE use and inventory at the level of the facility and the dialysis organization is advisable. Preserving and prioritizing PPE for health-care workers and patients is a challenge. Alternative solutions include recycling protocols, extended use of eye and face protective gear and alternative PPE production methods. The CDC has provided specific guidance for these options ${ }^{8}$.

\section{Management of the workforce}

Another crucial challenge is management of human resources. Reports from areas with high numbers of infected patients describe excessive mental and physical burden on health-care workers. Moreover, reports suggest that in Italy, nearly $10 \%$ of people with COVID-19 are health-care personnel ${ }^{9}$. Therefore, efficient utilization of the workforce along with appropriate planning for peak disease activity is crucial. Actions that can be taken include surveying health-care workers for specific skill sets, creating alternate schedules, limiting prolonged exposure to clinical areas and arranging social support networks. Most importantly, each unit should plan for an increase in disease activity and a decrease in workforce due to illness by creating a list of back-up staff for all positions.

\section{Facilitating home dialysis}

Planning for possible exhaustion of outpatient dialysis facilities should be done now. Home dialysis is an attractive alternative that combines dialysis with social distancing and elimination of transportation needs. Some dialysis organizations suggest facilitating home dialysis for as many patients with kidney failure as possible with timely placement of peritoneal catheters and vascular access. These surgical procedures should be considered urgent rather than elective as hospitals prioritize their surgical capabilities during the pandemic.

\section{Areas that require standardization}

Some areas exist in which the response to COVID-19 is not standardized across facilities, and additional data and guidance are required. For example, resource utilization, especially appropriate use of PPE in asymptomatic patients, has been hotly debated by the dialysis community and requires clear guidance from health-care authorities. Under conditions of limited COVID-19 testing capability, prioritizing testing of patients on dialysis for screening versus diagnosis and management (that is, 
recurrent testing to detect disappearance of the virus) is recommended. Substantial resource allocation including of test kits, sampling personnel and laboratory resources is required to accomplish widespread testing of patients on dialysis.

Transportation of patients with COVID-19 requires strict protocols and thoughtful preparation ${ }^{10}$. Drivers require training and protective equipment, but no current guidance is available for either commercial or private drivers. Hospitals receiving patients with confirmed or suspected COVID-19 should be notified and in the USA, CDC Interim Guidance for Emergency Medical Services Systems should be implemented.

The CDC has provided some guidance regarding return to work for health-care workers who have recovered from COVID-19 but these guidelines continue to evolve. A shortage of dialysis technicians and nurses already exists and a further decrease in the workforce could be catastrophic. Dialysis companies should consider creating fast-tracked training opportunities to provide additional health-care workers and a back-up system to ensure adequate staffing. The unavoidable economic impact of the pandemic has forced many people out of work, resulting in an unprecedented opportunity for recruitment.

Finally, regulatory agencies are currently considering temporary changes in patient care regulations such as allowing patients to swiftly move between health systems and changes in telehealth regulations. For example, coordination of dialysis resources from different providers should be facilitated to enable easy cohorting and management of patients with COVID-19.

The current situation due to the COVID-19 pandemic is unprecedented, but this crisis also provides a unique opportunity to gather vital information to process and learn from. With the advent of information technology and fast-paced communication platforms, we will undoubtedly be more prepared for the next challenge.
1. Coronavirus COVID-19 global cases by the Center for Systems Science and Engineering (CSSE) at Johns Hopkins University (JHU). JHU https://coronavirus.jhu.edu/map.html (2020).

2. Guan, W.-j. et al. Clinical characteristics of coronavirus disease 2019 in China. N. Engl. J. Med. https://doi.org/10.1056/NEJMoa2002032 (2020).

3. Onder, G., Rezza, G. \& Brusaferro, S. Case-fatality rate and characteristics of patients dying in relation to COVID-19 in Italy. JAMA https://doi.org/10.1001/jama.2020.4683 (2020).

4. Flahault, A. COVID-19 cacophony: is there any orchestra conductor? Lancet https://doi.org/10.1016/S0140-6736(20)30675-9 (2020).

5. Centers for Disease Control and Prevention. Interim guidance for infection prevention and control recommendations for patients with suspected or confirmed COVID-19 in outpatient hemodialysis facilities. CDC https://www.cdc.gov/coronavirus/2019-ncov/ healthcare-facilities/dialysis.html (2020).

6. American Society of Nephrology. Nephrologists Transforming Dialysis Safety (NTDS), Coronavirus Disease 2019. ASN https://www.asn-online.org/ntds/ (2020).

7. Cheng, V. C. C. et al. Escalating infection control response to the rapidly evolving epidemiology of the coronavirus disease 2019 (COVID-19) due to SARS-CoV-2 in Hong Kong. Infect. Control Hosp. Epidemiol. https://doi.org/10.1017/ice.2020.58 (2020).

8. Centers for Disease Control and Prevention. Strategies for optimizing the supply of PPE. CDC https://www.cdc. gov/coronavirus/2019-ncov/ hcp/ppe-strategy/index.html (2020).

9. CNN. Nearly 1 in 10 of Italy's infected are health care workers. CNN https://edition.cnn.com/world/live-news/coronavirus-outbreak03-22-20/h_e27a10efe9dfe61900b2ae6583e13189 (22 March 2020).

10. Nacoti, M. et al. At the epicenter of the Covid-19 pandemic and humanitarian crises in Italy: changing perspectives on preparation and mitigation. NEJM Catal. https://doi.org/10.1056/CAT.20.0080 (2020).

\section{Acknowledgements}

The authors thank D. Rodgers and S. Stark (both American Society of Nephrology) for their invaluable comments and suggestions.

\section{Competing interests}

T.A.I. is a member of the American Society of Nephrology (ASN) COVID-19 response Team and chairs the Nephrologists Transforming Dialysis Safety (NTDS) Current Emerging Threats Subcommittee. He reports the following disclosures unrelated to the article content: Fresenius Kabi, $\mathrm{GmbH}$, Abbott Renal Care and International Society of Nephrology for consulting work. A.S.K. is a member of ASN COVID-19 Response Team and chairs the NTDS initiative. He reports the following disclosures unrelated to the article content: consultancy agreements with the ASN and National Institutes of Diabetes and Digestive and Kidney Diseases; honorarium from several universities, medical schools and professional organizations, including honorarium for lectures, seminars and webinars; and scientific advisership and membership for Qualidigm. He also reports other interests and relationships with the ASN and Renal Physicians Association. 